\section{Pediatric Review - International Journal of Pediatric Research}

2021 Volume 8 Number 1 January-February

\title{
Scrub septicemia progressing to polyneuropathy: a case report
}

\section{Kumar Jena P. ${ }^{1}$, Kabi Satapathy S. ${ }^{2}$, Satya Prakash Nayak A. ${ }^{3}$, Nalik 5. ${ }^{4}$, Charan Murmu M. ${ }^{5 *}$ \\ DOI: https://doi.org/10.17511/ijpr.2021.i01.09}

\footnotetext{
1 Pradeep Kumar Jena, Associate Professor, Department of Pediatrics, SCB Medical College, Cuttack, Odisha, India.

2 Swasthi Kabi Satapathy, Resident Physician, Department of Pediatrics, SCB Medical College, Cuttack, Odisha, India.

${ }^{3}$ Alok Satya Prakash Nayak, Senior Resident, Department of Pediatrics, SCB Medical College, Cuttack, Odisha, India.

${ }^{4}$ Sarthak NaIik, Senior Resident, Department of Pediatrics, SCB Medical College, Cuttack, Odisha, India.

5* Mangal Charan Murmu, Associate Professor, Department of Pediatrics, SCB Medical College, Cuttack, Odisha, India.
}

Scrub typhus is a major public health problem in India with a wide spectrum of clinical manifestations and a low index of suspicion is low endemic areas which in turn can increase morbidity and mortality. The clinical manifestations of scrub typhus can range from mild non-specific febrile illness or constitutional symptoms such as fever, rash, myalgia, headache to various organ dysfunction such as AKI, myocarditis, pneumonia, meningitis, encephalitis, GI bleed, hepatitis. Here we report a case of a 9-year-old girl who presented with scrub septicemia and progressed to develop critical illness myopathy, which is very unusual.

Keywords: Scrub Typhus, Myopathy, Polyneuropathy

Corresponding Author

Mangal Charan Murmu, Associate Professor, Department of Pediatrics, SCB Medical College, Cuttack, Odisha, India.

Email: mangal74murmu@yahoo.co.in
How to Cite this Article

To Browse

Jena PK, Satapathy SK, Nayak ASP, NaIik S, Murmu

MC. Scrub septicemia progressing to polyneuropathy: a case report. Pediatric Rev Int J Pediatr Res. 2021;8(1):64-67.

Available From

https://pediatrics.medresearch.in/index.php/ijpr/arti cle/view/652

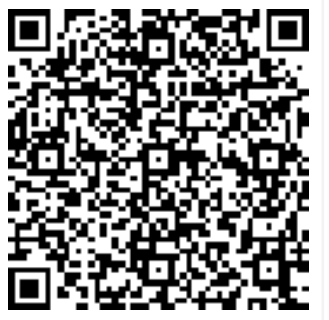

Manuscript Received 2021-01-06

Conflict of Interest No

Review Round 1
2021-01-16
Funding
Nil

Review Round 1 $\mathrm{Nil}$

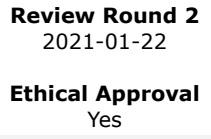

Review Round 3

Accepted 2021-01-27

Plagiarism X-checker $9 \%$

Note

(c) 2021 by Pradeep Kumar Jena, Swasthi Kabi Satapathy, Alok Satya Prakash Nayak, Sarthak NaIik, Mangal Charan Murmu and Published by Siddharth Health Research and Social Welfare Society. This is an Open Access article licensed under a Creative Commons Attribution 4.0 International License https://creativecommons.org/licenses/by/4.0/ unported [CC BY 4.0].

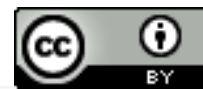




\section{Introduction}

Scrub typhus is an acute febrile illness that is caused by mite borne Orientia Tsutsugamushi. At present it presents as a major public health problem in India with a large number of cases being reported from various states and Union territories. It possesses major public health concern due to its wide spectrum of clinical manifestations and low index of suspicion is low endemic areas which in turn can increase morbidity and mortality. The clinical manifestations of scrub typhus can range from mild non-specific febrile illness or constitutional symptoms such as fever, rash, myalgia, headache to various organ dysfunction such as AKI, myocarditis, pneumonia, meningitis, encephalitis, GI bleed, hepatitis [1-4].

Critical illness myopathy is a syndrome of widespread muscle weakness and neurological dysfunction which can develop in critically ill patients receiving intensive care. Here we report a case of a 9-year-old girl who presented with scrub septicemia and progressed to develop critical illness myopathy.

\section{Case Review}

A 9-year-old female child was admitted to our hospital, referred from another tertiary care centre of the state with a history of high-grade fever ( $>101$ degrees $F$ ) for 7 days associated with multiple episodes of vomiting and 1 episode of GTCS type of convulsion on day 5-6 of fever. The patient was treated at the previous centre for 2 days where she had developed hypotensive shock and respiratory distress.

At the time of admission to our institution patient had a temperature of 100.6 degrees F, respiratory rate of $34 / \mathrm{min}$, heart rate of $120 / \mathrm{min}$, Spo2 $95 \%$ with $\mathrm{O} 2$ inhalation through NRM. Blood pressure was maintained at $106 / 62 \mathrm{mmHg}$ with vasopressors.

She had pallor, hepatosplenomegaly and coarse crepitations were heard in all areas of auscultation. The patient had a GCS of $13 / 15$, brisk DTRs, positive Babinski sign, and positive meningeal signs. Fundoscopy revealed signs of papilledema. She was started on IV antibiotics, hypertonic saline infusion, anticonvulsants and was continued on vasopressors. Because of the high endemicity of Scrub typhus in this region she was empirically started on IV Doxycycline.
Samples were collected and sent for electrolytes, Scrub typhus ELISA, MP-ICT and blood culture and sensitivity. Scrub typhus Ig-M came positive. Doxycycline was continued. Serum sodium levels were 151 . The hypertonic saline infusion was omitted and the patient was started on IV mannitol.

6 hours of post-admission patient developed gallop rhythm with increasing respiratory distress. Anti failure measures were taken. The patient was shifted to PICU around 14 hours post-admission. ABG parameters showed a $\mathrm{PaO2}$ of 101 with a $\mathrm{PaO} 2 /$ FiO2 ratio of $<200$, PCO2 27, AADO2 $145, \mathrm{pH}$ 7.438. The patient was intubated and put on a mechanical ventilator because of impending respiratory failure.

Chest Xray on Day 2 revealed $B / L$ non-homogenous patchy opacities. Lumbar puncture was performed which revealed features of pyogenic meningitis with 12 cells - $60 \%$ being polymorphonuclear, sugar $66 \mathrm{mg} / \mathrm{dl}$, protein $=154 \mathrm{mg} / \mathrm{dl}$. CSF viral marker study came negative for JEIgM as well as HSV. A provisional diagnosis of Scrub encephalitis with ARDS was made.

After 48 hours of admission and nearly 32 hours post ventilation patient was maintaining off vasopressors and on minimal ventilator settings. ABG parameters improved. $\mathrm{PaO} 2$ was $140, \mathrm{PaCO} 2$ $18, \mathrm{pH} 7.36, \mathrm{HCO}-15, \mathrm{AaDO} 2$ 63. The patient was extubated. Her sensorium gradually improved. On post extubating day 2 she developed left lower lobe consolidation. She was started on N acetylcysteine nebulization along with chest physiotherapy. Antibiotics were upgraded.

On postintubation day 3 patient developed hypernatremia which was corrected with extra fluid administration and simultaneous monitoring for signs of fluid overload. Post extubating patient gradually developed diminishing muscle power, absent deep tendon reflexes and hypotonia. Power on day 3 of illness - left upper $\operatorname{limb}=3 / 5$, right upper $\operatorname{limb}=3 / 5$, left lower $\operatorname{limb}=1 / 5$, right lower $\operatorname{limb}=1 / 5$.

There was no associated muscle tenderness, no progression of flaccid paralysis, no respiratory or bulbar involvement.

A differential of GBS / CIM /Scrub myopathy was suspected. A nerve conduction study and EMG was planned. On Day 7 of onset of flaccid paralysis NCS and EMG were performed. NCS revealed features suggestive of motor axonal neuropathy. 
EMG suggested a myopathic pattern ( small amplitude, short duration, early recruitment polyphasic motor unit action potentials). Serum Creatinine kinase level was elevated ( 2302 IU/I)

A diagnosis of Critical illness polyneuropathy myopathy was made secondary to multisystem involvement due to scrub septicemia. The patient's muscle power gradually improved over 2 weeks and discharged successfully with advice for physiotherapy.

\section{Discussion}

Critical illness myopathy, critical illness polyneuropathy and the overlap CIPNM are amongst the most common causes of neuromuscular weakness in the intensive care setting. The major risk factors for this include severe sepsis, SIRS, multiple organ failure. Other risk factors include -

- increased duration of hospital stay

- hyperglycemia

- electrolyte imbalance

- female gender

- severity of illness

- duration of organ dysfunction

- renal failure and renal replacement therapy

Low serum albumin

- parenteral nutrition

- hyperosmolarity

- vasopressor, catecholamine and high-grade corticosteroid use

- septic encephalopathy

Here we report a case of ICU acquired weakness most likely due to critical illness myopathy polyneuropathy overlap. Incidence of such illness due to scrub septicemia has not been reported or is unknown. The development of acute flaccid paralysis in this patient raised the suspicion of Guillain Barre syndrome however the timing of onset of the quadriparesis, lack of progression, presence of flexor plantar response and the electrodiagnostic studies were suggestive against this diagnosis.

Critical illness polyneuropathy is characterized by low-amplitude compound motor action potentials with normal or slightly reduced nerve conduction velocities, findings characteristic of axonal neuropathy.
Critical illness myopathy may be associated with loss of muscle responsiveness to direct stimulation or with "myopathic" abnormalities (low-amplitude, polyphasic motor unit action potentials with early recruitment) or maybe normal. As in this case both findings co-exist a diagnosis of CIPNM was made. There is no published data on the exact incidence of CIP/CIM in children. The only prospective study to date estimated an incidence of $1.7 \%$, which is much lower than in adults $[1,2]$. This issue has not been systematically studied in pediatric ICUs except above mentioned prospective study where ICUAW was found in 14 children mostly pediatric transplant patients (post bone marrow or solid organ transplant) with sepsis ( 9 children) and multi-organ dysfunction (11 children) out of 830 patients admitted to pediatric ICU [3].

The lack of much available data on this entity in children could be due to failure to identify the problem in the pediatric population. In children with CIP/CIM, the cases described so far have been identified because of muscle weakness and failure to wean from the ventilator. The timing of onset of weakness was variable. In some cases, it was very early in the onset of critical illness [4,5]. In the only prospective study done so far, weakness was clinically noticed after a wide range of duration of illness (4-26 days) and $86 \%$ of these children were mechanically ventilated for $>5$ days [1].

Due to the unavailability of any specific diagnostic criteria paediatricians and intensivists need to have a high index of suspicion for CIP/CIM in the paediatric population. Diagnosis is usually made after the exclusion of other known causes of muscle weakness. Spontaneous recovery occurs within weeks in mild cases, and in months in moderate to severe cases, or does not occur at all in some of the cases. [6]

This case signifies the severity of infection due to scrub typhus which is an emerging public health concern. Our patient possessed several risk factors for developing CIPNM such as multiple organ dysfunction, ARDS, female sex, electrolyte imbalance, corticosteroid use, use of sedatives, duration of illness $>10-12$ days. Since there are no proven strategies that prevent or treat CIM/CIP it is of utter importance to treat the underlying systemic illness promptly. In this case timely initiation of IV Doxycycline and early weaning off vasopressors and sedatives could have resulted in preventing progression to profound weakness and a greater degree of muscle damage. 
ICU acquired weakness can cause difficulty in weaning from ventilators as well as a significant increase in morbidity and mortality. Diagnosis is possible with clinical suspicion, electrophysiologic studies and confirmed by muscle biopsy testing. However, unfortunately, our knowledge about the disease condition in the pediatric population is quite limited. More studies are needed to better identify the risk factors in the pediatric population and the natural history of the disease in children.

\section{Reference}

01. Barnwell BL, Mildner RJ, Hassall AC, Becker LE, Vassar J, Shemie SD. Muscle weakness in critically ill children. Neurology. 2003;61(12)1779-82.

doi: $\quad 10.1212 / 01 . w n l .0000098886 .90030 .67$ [Crossref]

02. Williams S, Horrocks IA, Ouvrier RA, Gillis J, Ryan MM. Critical illness polyneuropathy and myopathy in pediatric intensive care- A review. Pediatr Crit Care Med. 2007;8(1)18-22.

doi: $\quad 10.1097 / 01 . p c c .0000256623 .01254 .40$

[Crossref]

03. Vinay Kukreti, Mosharraf Shamim, Praveen Khilnani. Intensive care unit acquired weakness in children- Critical illness polyneuropathy and myopathy. Indian J Crit Care Med. 2014;18(2)95-101.

doi: $10.4103 / 0972-5229.126079$ [Crossref]

04. Sheth RD, Pryse-Phillips WE, Riggs JE, Bodensteiner JB. Critical illness neuromuscular disease in children manifested as ventilatory dependence. J Pediatr. 1995;18(2)95-101. doi: $10.4103 / 0972-5229.126079$ [Crossref]

05. De Jonghe B, Sharshar T, Lefaucheur JP, Authier FJ, Durand-Zaleski I, Boussarsar $M$, et al. Paresis acquired in the intensive care unit- $A$ prospective multicenter study. JAMA. $2002 ; 288 ; 2859-67$.

doi: $10.1001 /$ jama.288.22.2859 [

Crossref ]

06. Latronico N, Peli E, Botteri M. Critical illness myopathy and neuropathy. Curr Opin Crit Care. $2005 ; 11(2) 126-32$.

doi: $\quad 10.1097 / 01 . c c x .0000155357 .24360 .89$

[Crossref] 